\title{
Posterior Tibialis Muscle
}

National Cancer Institute

\section{Source}

National Cancer Institute. Posterior Tibialis Muscle. NCI Thesaurus. Code C140526.

A muscle of the lower leg that is located in the deep posterior compartment, originating on the inner posterior borders of the tibia and fibula, with further attachments to the interosseous membrane; the tendon of the posterior tibialis muscle, the posterior tibial tendon, terminates by dividing into plantar, main and recurring components, all of which have terminal insertions: plantar portion inserts into the bases of the second, third and fourth metatarsals; the intermediate and lateral cuneiforms, and the cuboid; the main portion inserts into the tuberosity of the navicular and the plantar surface of the medial cuneiform; the recurrent portion inserts into the sustentaculum tali of he calcaneus. The muscle functions to stabilize the ankle, as well as invert and plantar flex the foot at the ankle. 